01

\title{
Термодинамические свойства фуллерита $\mathbf{C}_{70}$
}

\section{() С.Ш. Рехвиашвили}

Институт прикладной математики и автоматизации КБНЦ РАН, Нальчик

E-mail: rsergo@mail.ru

Поступило в Редакцию 2 мая 2017 г.

В рамках квантово-статистического метода получены новое выражение для изохорной теплоемкости и уравнение состояния фуллерита $\mathrm{C}_{70}$. Выведены аналоги законов Дебая и Дюлонга-Пти. Молекулы $\mathrm{C}_{70}$ моделируются квантовыми изотропными осцилляторами в предположении, что их несферичность слабо влияет на термодинамические свойства конденсированной фазы. Внутримолекулярные колебания атомов углерода описываются теорией теплоемкости Дебая, холодный вклад в свободную энергию вычисляется с использованием парного потенциала Леннарда-Джонса для молекул фуллерена. Сравнение теории с экспериментом показывает хорошее согласие.

DOI: 10.21883/PJTF.2017.16.44931.16832

Молекула фуллерена $\mathrm{C}_{70}$ получается из $\mathrm{C}_{60}$ путем растяжения и введения в экваториальную область дополнительного кольца, состоящего из десяти атомов углерода $[1,2]$. При этом молекула приобретает форму вытянутого сфероида высотой $0.78 \mathrm{~nm}$ и диаметром $0.694 \mathrm{~nm}$. Ввиду указанной асимметрии структура твердой (кристаллической) фазы $\mathrm{C}_{70}$ существенно зависит от взаимной ориентации молекул. Здесь обнаруживаются ГЦК- и ОЦК-структуры, а также моноклинная, ромбическая и гексагональная сингонии [3]. Вращательная динамика молекул $\mathrm{C}_{70}$ и ее влияние на физические и структурные свойства твердой фазы фуллерита изучались в [4-7]. Было, в частности, отмечено, что в зависимости от температуры и давления имеют место одноосное и квазисвободное вращение молекул. Помимо вращения возможны также либрационные колебания молекул.

Чтобы рассчитать термодинамические свойства фуллерита $\mathrm{C}_{70}$ в широких диапазонах температур и давлений, необходимо рассмотреть ансамбль молекул $\mathrm{C}_{70}$ с учетом пространственной анизотропии потен- 
циала взаимодействия (в общем случае имеется ориентационная зависимость), колебательно-вращательной и внутримолекулярной динамики, что представляется чрезвычайно сложной задачей. Упрощенный подход к расчету термодинамических свойств фуллерита $\mathrm{C}_{70}$ основывается на предположении об эффективном радиусе молекул $(R=0.38 \mathrm{~nm})[8]$. В этом случае фактически предполагается, что в составе твердой фазы молекулы $\mathrm{C}_{70}$ вращаются изотропно и это вращение не влияет на потенциальную энергию взаимодействия молекул и термодинамические свойства вещества. Формой молекул также пренебрегается при самосогласованном определении параметров парного межмолекулярного потенциала для различных фуллеренов [9] и в методе молекулярнодинамического моделирования [10]. В настоящей работе в рамках теоретической модели $[11,12]$ и в приближении эффективного радиуса молекул проводится расчет термодинамических свойств фуллерита $\mathrm{C}_{70}$.

Молекулы $\mathrm{C}_{70}$ представляются в виде квантовых изотропных осцилляторов, совершающих колебательно-вращательное движение; внутримолекулярные тепловые колебания атомов углерода описываются в рамках теории Дебая [11]. Свободная энергия фуллерита складывается из потенциальной энергии $U$, колебательно-вращательного $F_{1}$ и внутримолекулярного $F_{2}$ вкладов; холодная составляющая свободной энергии $U$, обусловленная силами притяжения и отталкивания между молекулами фуллерена, вычисляется в континуальном приближении с использованием потенциала Леннарда-Джонса [12]. Раздельное описание колебательно-вращательных и внутримолекулярных мод соответствует адиабатическому приближению. Предполагается также, что при всестороннем сжатии представление об изотропных осцилляторах остается применимым. Вычисления в рамках квантово-статистической модели $[11,12]$ для одного моля фуллерита $\mathrm{C}_{70}$ приводят к следующим результатам.

1. Изохорная теплоемкость

$$
C_{V}=C_{V 1}+C_{V 2},
$$

где

$$
C_{V 1}=9 R y_{1}^{2} \int_{0}^{1} \frac{\left[\exp \left(-2 x y_{1}\right)+6 \exp \left(-x y_{1}\right)+1\right] \exp \left(-x y_{1}\right) x^{4} d x}{\left[1-\exp \left(-x y_{1}\right)\right]^{2}\left[1+\exp \left(-x y_{1}\right)\right]^{2}},
$$

Письма в ЖТФ, 2017, том 43, вып. 16 


$$
\begin{gathered}
C_{V 2}=630 R y_{2}^{2} \int_{0}^{1} \frac{\exp \left(-x y_{2}\right) x^{4} d x}{\left[1-\exp \left(-x y_{2}\right)\right]^{2}}, \\
y_{i}=\frac{\theta_{i}}{T}\left(\frac{V_{0}}{V}\right)^{\gamma_{i}} \quad(i=1,2) .
\end{gathered}
$$

Здесь $R$ - универсальная газовая постоянная, $T-$ абсолютная температура, $\theta_{i}$ и $\gamma_{i}$ - характеристические температуры и аналоги параметра Грюнайзена, отвечающие колебательно-вращательной $(i=1)$ и внутримолекулярной $(i=2)$ модам, $V-$ равновесный объем, $V_{0}-$ равновесный объем, соответствующий минимуму потенциальной энергии при $T=0$. Для низких температур при $y_{i} \gg 1$ и $y_{1} \ll y_{2}$ получаем аналог закона Дебая

$$
C_{V}=3 \pi^{4} R\left(\frac{9}{10 y_{1}^{3}}+\frac{56}{y_{2}^{3}}\right) \approx \frac{27}{10} \pi^{4} R\left(\frac{T}{\theta_{1}}\right)^{3} .
$$

Для высоких температур при $y_{i} \ll 1$ из (1) следует аналог закона Дюлонга-Пти

$$
C_{V}=216 R \approx 1.796 \cdot 10^{3} \mathrm{~J} /(\mathrm{K} \cdot \mathrm{mol}) .
$$

2. Уравнение состояния

$$
p=\frac{B}{2}\left[\left(\frac{V_{0}}{V}\right)^{5}-\left(\frac{V_{0}}{V}\right)^{3}\right]+\frac{\gamma_{1} E_{1}+\gamma_{2} E_{2}}{V},
$$

где

$$
\begin{gathered}
E_{1}=9 R \theta_{1}\left[\frac{3}{8}-\int_{0}^{1} \frac{\exp \left(-x y_{1}\right)\left(3 \exp \left(-x y_{1}\right)+1\right) x^{3} d x}{\exp \left(-2 x y_{1}\right)-1}\right], \\
E_{2}=630 R \theta_{2}\left(\frac{1}{8}-\int_{0}^{1} \frac{\exp \left(-x y_{2}\right) x^{3} d x}{\exp \left(-x y_{2}\right)-1}\right),
\end{gathered}
$$

$B$ - объемный модуль упругости.

Были проведены численные расчеты по (1), (2) и сравнение результатов этих расчетов с экспериментальными данными работ $[13,14]$. Равновесный объем при нулевой температуре рассчитывался через

Письма в ЖТФ, 2017, том 43, вып. 16 


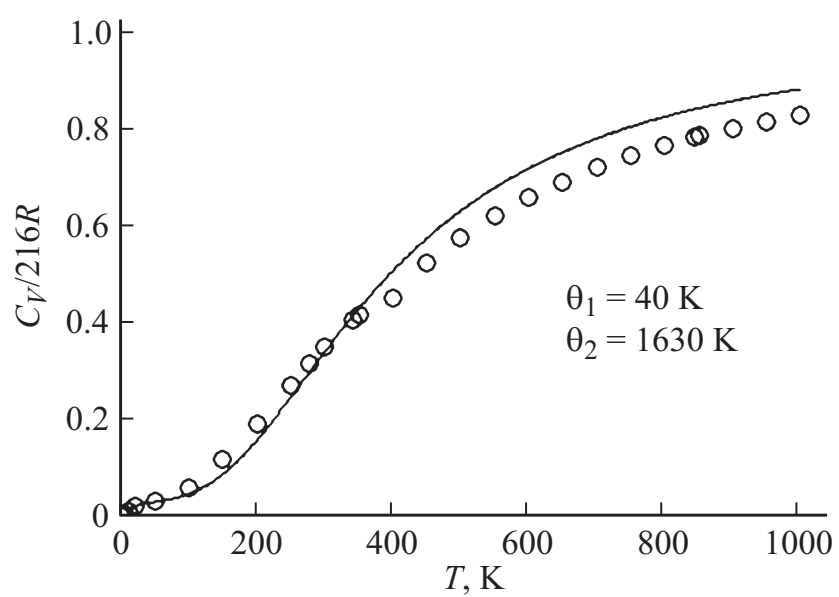

Рис. 1. Температурная зависимость изохорной теплоемкости фуллерита $\mathrm{C}_{70}$.

молярную массу и плотность фуллерита $V_{0}=M / \rho=4.94 \cdot 10^{-4} \mathrm{~m}^{3} / \mathrm{mol}$. Характеристические температуры соответствовали фуллериту $\mathrm{C}_{60}$ [11]: $\theta_{1}=40 \mathrm{~K}$ и $\theta_{2}=1630 \mathrm{~K}$. Выбор этих значений для фуллерита $\mathrm{C}_{70}$ обусловлен применением приближения эффективного радиуса молекул. Для объемного модуля упругости и параметра Грюнайзена, отвечающего за внутримолекулярный вклад, приняты максимальные значения из работ $[14,15]: B=34 \mathrm{GPa}, \gamma_{2}=0.226$.

На рис. 1 результаты расчета изохорной теплоемкости по формуле (1) при $V=V_{0}$ (сплошная линия) сравниваются с данными (кружки), найденными с учетом экспериментальных значений изобарной теплоемкости, температурного коэффициента объемного расширения и изотермической сжимаемости, которые приведены в [13]. Можно видеть, что достаточно хорошее согласие между теорией и экспериментом достигается во всем диапазоне температур. Отметим, что в области температур $30-50 \mathrm{~K}$ происходит относительная стабилизация теплоемкости вблизи $6 R[13]$. Данное значение изохорной теплоемкости точно получается в рамках настоящей модели: $C_{V 1}=6 R$ при $T \rightarrow \infty$. При $T>0.1 \theta_{2}$ доминирующим становится вклад, обусловленный внутримолекулярными колебаниями атомов углерода.

Письма в ЖТФ, 2017, том 43, вып. 16 


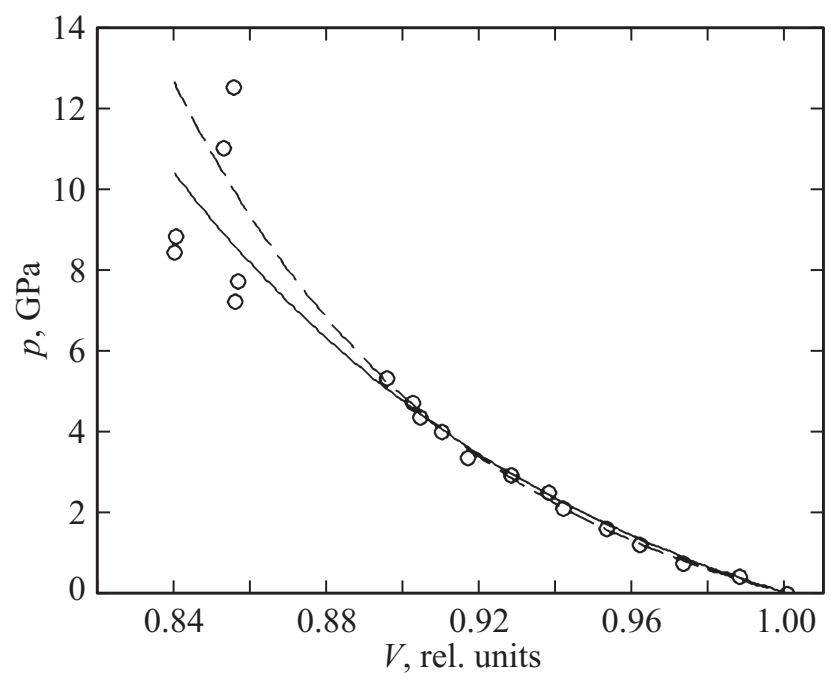

Рис. 2. Изотерма сжатия фуллерита $\mathrm{C}_{70}$ : сплошная кривая - уравнение (2), штриховая - уравнение Мурнагана, кружки - экспериментальные данные.

На рис. 2 представлены результаты расчета по уравнению состояния (2) при $T=293 \mathrm{~K}$ в сравнении с экспериментальными данными и результатами расчета по уравнению состояния Мурнагана из работы [14]. Параметр $\gamma_{1}$ варьировался для получения наилучшего согласования расчетов с экспериментальными данными. Таким образом было получено значение $\gamma_{1}=7.138$. При заданных численных значениях всех параметров уравнение $p=0$ имеет приближенное решение: $V / V_{0} \approx 1.023$, которое учитывалось при построении графика. Проведенные расчеты достаточно хорошо согласуются с экспериментальными данными: средняя относительная погрешность, вычисленная по всем точкам, составляет $\sim 5 \%$. Для уравнения состояния Мурнагана эта погрешность достигает 10\%. Расчеты показывают, что для фуллерита $\mathrm{C}_{70}$ выполняется условие $\gamma_{1}>\gamma_{2}$. В работе [12] для фуллерита $\mathrm{C}_{60}$ на основе численных расчетов было принято $\gamma_{1}=\gamma_{2}$. Однако если один параметр считать известным и в расчетах использовать его максимальное экспериментальное значение $\gamma_{2}=0.23$ [16], то для другого параметра в результате оптимизации получается значение $\gamma_{1}=0.358$, т. е. приходим 
к выводу, что условие $\gamma_{1}>\gamma_{2}$ для фуллерита $\mathrm{C}_{60}$ также выполняется. Обратим, кроме того, внимание на то, что $\gamma_{1}\left(\mathrm{C}_{70}\right)>\gamma_{1}\left(\mathrm{C}_{60}\right)$. Физически то означает, что у фуллерита $\mathrm{C}_{70}$ колебательно-вращательная динамика молекул заметнее сказывается на термодинамических свойствах, чем у фуллерита $\mathrm{C}_{60}$.

Таким образом, в работе показано, что квантово-статистическая модель, предложенная в $[11,12]$ для фуллерита $\mathrm{C}_{60}$, в целом успешно описывает термодинамические свойства фуллерита $\mathrm{C}_{70}$ при одних и тех же значениях характеристических температур $\theta$. Из этого следует, что несферичность молекул $\mathrm{C}_{70}$ не оказывает критического влияния на макроскопические свойства кристаллической фазы. Этот вывод представляется вполне естественным для достаточно широких интервалов температур и давлений, когда имеет место квазисвободное вращение молекул.

\section{Список литературы}

[1] McKenzie D.R., Davis C.A., Cockayne D.J.H. et al. // Nature. 1993. V. 355. P. 622.

[2] Елеикий А.В., Смирнов Б.М. // УФН. 1993. Т. 163. № 2. С. 33.

[3] Verheijen M.A., Meekes H., Meijer G. et al. // Chem. Phys. 1992. V. 166. P. 287.

[4] Maniwa Y., Ohi A., Mizoguchi K. et al. // J. Phys. Soc. Jpn. 1993. V. 62. P. 1131.

[5] Soldatov A., Sundqvist B. // J. Phys. Chem. Solids. 1996. V. 57. N 9. P. 1371.

[6] Thirunavukkuarasu K., Long V.C., Musfeldt J.L. et al. // J. Phys. Chem. 2011. V. 115. N 9. P. 3646.

[7] Смирнов В.П., Зубок Д.А. // ФТТ. 1997. Т. 39. В. 10. С 1895.

[8] Kniaz K, Girifalco L.A., Fischer J.E. // J. Phys. Chem. 1995. V. 99. N 45. P. 16804.

[9] Магомедов М.Н. // ФТТ. 2005. Т. 47. В. 4. С. 758.

[10] Хуснутдинов Р.М., Мокшин А.В., Тахавиев И.Д. // ФТТ. 2015. Т. 57. В. 2. C. 393.

[11] Рехвиашвили С.Ш. // ФТТ. 2013. Т. 55. В. 7. С. 1422.

[12] Рехвиашвили С.Ш. // ФТТ. 2017. Т. 59. В. 4. С. 816.

[13] Дикий В.В., Кабо Г.Я. // Успехи химии. 2000. Т. 69. В 2. С. 107.

[14] Christides C., Thomas I.M., Dennis T.J.S., Prassides K. // Europhys. Lett. 1993. V. 22. P. 611.

[15] Мелетов К.П., Максимов А.А., Тартаковский И.И. // ЖЭТФ. 1997. Т. 111. B. 1. C. 262 .

[16] Мелетов К.П., Kourouklis G., Christofilos D., Ves S. // ЖЭТФ. 1995. Т. 108. B. 4. C. 1456 .

Письма в ЖТФ, 2017, том 43, вып. 16 